livraisons

d'Histoire

de l'Architecture

\section{Livraisons de l'histoire de l'architecture}

32 | 2016

Les représentations de l'architecture

\title{
La distorsion. Un mode de représentation médiévale de l'architecture et ses épigones modernes
}

The distortion

Verzerrung. Architekturdarstellung im Mittelalter und ihre neuzeitlichen

Epigonen

Nicolas Reveyron

\section{OpenEdition}

\section{Journals}

Édition électronique

URL : http://journals.openedition.org/lha/630

DOI : $10.4000 /$ /ha. 630

ISSN : 1960-5994

Éditeur

Association Livraisons d'histoire de l'architecture - LHA

Édition imprimée

Date de publication : 31 décembre 2016

Pagination : 9-20

ISSN : 1627-4970

\section{Référence électronique}

Nicolas Reveyron, « La distorsion. Un mode de représentation médiévale de l'architecture et ses épigones modernes », Livraisons de l'histoire de l'architecture [En ligne], 32 | 2016, mis en ligne le 31 décembre 2018, consulté le 19 avril 2019. URL : http://journals.openedition.org//ha/630 ; DOI : 10.4000//ha. 630 
Par Nicolas ReVEYron

\section{LA DISTORSION. UN MODE DE REPRÉSENTATION MÉDIÉVAL DE L'ARCHITECTURE ET SES ÉPIGONES MODERNES}

Les représentations médiévales de l'architecture s'inscrivent dans un très large éventail de modalités, allant de la figuration réaliste à la désagrégation du modèle. Cette extraordinaire diversité, qui, dans ses concomitances et par ses audaces, semble préfigurer l'art du $\mathrm{XX}^{\mathrm{e}}$ siècle, répond à des intentions et des significations associant recherches esthétiques, orientations culturelles et pensée spécifique de l'architecture. Dans ce dernier cas, l'approche anthropologique de la question s'appuie sur un corpus littéraire éclairant ${ }^{1}$. Si l'art gothique tend rapidement au réalisme, plus exactement à des formes de vérisme ${ }^{2}$, les figurations romanes de l'architecture interprètent plus librement leur modèle ; peut-on d'ailleurs parler de modèle, tant l'édifice référenciel n'est, le plus souvent, rattaché à l'idée directrice de l'œuvre que par quelques détails signifiants, selon un processus similaire à celui que Richard Krautheimer a mis en évidence pour l'architecture elle-même ${ }^{3}$ ? Cette liberté d'interprétation est très large. Elle s'étend de ce qu'on appellerait spontanément, mais à tort, une maladresse figurative, jusqu'à ce phénomène de déconstruction-reconstruction qui trouve son origine dans l'art tardo-antique. Avec la figuration romane de l'archi-

1. Dans la vaste bibliographie sur la question, voir par exemple : Alain Labbé, L'Architecture des palais et des jardins dans les chansons de geste, Paris-Genève, 1987 ; Noël Duval, "Les descriptions d'architecture et de décor chez Grégoire de Tours et les auteurs gaulois : le cas de Saint-Martin de Tours ", La Naissance de la ville chrétienne: mélanges en hommage à N. Gauthier, B. Beaujard dir., Tours, Université de Tours, 2002, p. 21-58; Gaëlle Herbert de la Portbarre, Descriptions monumentales et discours sur l'édification chez Paulin de Nole, le regard et la lumière, Leiden-Boston, Brill, 2006; Marie-Christine Fayant, Description de sainte Sophie de Paul le Silentiaire, Paris, Université Paris XNanterre-CNRS, 2008 (Collection Textes, histoire et monuments de l'Antiquité au Moyen Âge) ; Didier Mehu, «Construction de mots, de figures et de pierres, L'exemple de la cathédrale de Chartres au temps de Fulbert ", Ad libros! Mélanges d'études médiévales offerts à Denise Angers et Joseph-Claude Poulin, Jean-François Cottier, Martin Gravel et Sébastien Rossignol dir., Montréal, Presses universitaires de Montréal, 2010, p. 83-103; Didier Mehu, "Les figures de l'édifice ecclésial d'après le Guide du pèlerin de Saint-Jacques de Compostelle ", Lieux sacrés et espace ecclésial (IXe-XVe siècle), Toulouse, Cahiers de Fanjeaux, n 46, éd. Privat, 2011, p. 80-113; Carlo Tosco, "Raoul Glaber et l'architecture ", Mélanges d'histoire de l'art offert à Xavier Barral i Altet, G. Mallet, V. Lucherini et M. Giannandrea dir., Paris, Picard, 2012, p. 372-383.

2. Il est évident que ces considérations préliminaires mériteraient d'être fortement nuancées, mais elles dépasseraient alors le cadre de cet article.

3. Richard Krautheimer, "Introduction to an "Iconography of mediaeval architecture" ", Journal of the Warburg and Courtauld Institutes, vol. 5, 1942, p. 1-33 (édition française : Introduction à une "Iconographie de l'architecture médiévale », Paris, éd. Gérard Montfort, 1993). 
tecture, nous entrons en effet dans le temps long de l'histoire de l'art. Le présent travail n'a pas pour ambition d'établir la chronologie ou la géographie d'un phénomène diversement caractérisé dans le temps et dans l'espace. Il cherche plutôt à mettre en évidence les mécanismes par lesquels l'art roman a traité l'image de l'architecture pour créer des compositions complexes, mais hautement signifiantes ${ }^{4}$. Il n'abordera donc que quelques exemples éclairants pris dans le corpus beaucoup plus vaste d'une recherche en cours.

\section{Aplatissement et restructuration de l'architecture figurée}

L'art romain classique et l'Antiquité tardive ont fourni des types de codifications formelles mis en ouvre encore dans les figurations romanes ${ }^{5}$. Comme l'a montré Ranuccio Bianchi Bandinelli, le glissement insensible de la figuration «illusionniste " élaborée dans la Grèce classique, et diversifiée par l'art hellénistique, à la représentation "non-réaliste ", qui est déjà bien affirmée dans l'art tardo-antique, intervient très tôt ${ }^{6}$. Il passe par le double mouvement d'un aplatissement de l'image et d'une restructuration non-réaliste de l'espace figuré. La représentation de la cavalcade ornant la base de la colonne antonine (161 ap. J.-C.), à Rome, est un exemple précoce d'aplatissement de la figuration par superposition des différents plans (cavaliers du premier plan, fantassins du second plan, cavaliers du troisième plan), tous traités avec la même précision (abandon de la perspective par estompage progressif) et les mêmes dimensions (abandon de la perspective par diminution progressive des figures).

Les nécessités de la communication politique ont vite accentué cette évolution. Ainsi, le bas-relief, aujourd'hui au musée de Tripoli, représentant le défilé de l'empereur sur l'arc de Septime Sévère à Leptis Magna (203 ap. J.-C.) montre le char impérial et les chevaux de profil, c'est-à-dire suivant le sens directionnel des personnages et du bas-relief, mais figure l'empereur et ses fils de face, c'est-à-dire en position de majesté, sans que cette incohérence spatiale gêne la lecture de l'image. De fait, le glissement d'une esthétique à l'autre s'avère particulièrement subtile, parce que l'aplatissement et la restructuration " anti-réalistes » de l'image coïncident avec une pérennité du réalisme dans la figuration des corps et des visages. Dans l'art roman, en revanche, la représentation " anti-réaliste " des personnages rejoint celle du cadre architectural.

4. Le présent travail fait suite à une première approche de la question présentée au colloque de Parme Medioevo: Immagine e memoria. Nicolas Reveyron, "L'image romane et la construction de la mémoire. L'exemple de la miniature figurant la Consécration de l'autel majeur de Cluny III par Urbain II en 1095 (Paris, BnF, ms 17716, $\mathrm{f}^{\circ} 91 \mathrm{r}^{\circ}$ ). Essai d'analyse iconique ", Medioevo : Immagine e memoria, Atti del XI Convegno Internazionale di studi di Parma (Parma 23-28 settembre 2008), A. C. Quintavalle dir., Milan, Electa, 2009, p. 185-193.

5. Tardo antico e alto Medioevo, La forma artistica nel passagio dall'Antichita al Medioevo, Atti del Convegno Internazionale (Roma 4-7 apr. 1967), Rome, Accademia Nazionale dei Lincei, 1968.

6. Ranuccio Bianchi Bandinelli, Dall'elenismo al medioevo, Rome, 1978. 
Au Moyen Âge central, c'est le tympan du portail, vaste page de communication ecclésiologique et théologique, qui bénéficie des formules les plus complexes de restructuration de l'espace. Figuration redressée à la verticale d'une image pensée originellement à l'horizontale, comme le prouvent la composition de celui de Moissac $^{7}$, il superpose strictement les registres, sans que cette disposition ne porte de signification : l'essentiel réside dans la latéralisation - à dextre et à senestre qui détermine le côté des élus et celui des damnés. Les jugements derniers des XII ${ }^{\mathrm{e}}$ XIII ${ }^{\mathrm{e}}$ siècles, par exemple, disposent de bas en haut la résurrection des morts, le paradis et l'enfer, les apôtres-assesseurs, les Quatre Vivants et le Christ-juge, entourés des anges installés dans les archivoltes. Or, le paradis est situé à la hauteur de l'enfer, les symboles des évangélistes devraient entourer le Christ sur un même plan horizontal et les anges des archivoltes ne peuvent dominer le Fils de Dieu : ils forment en réalité une assemblée qui l'entoure sur un autre plan horizontal, en position hiérarchiquement inférieure.

\section{Déconstruction-reconstruction}

Ce procédé de restructuration de l'espace qui préside à la composition du jugement dernier équivaut donc à la déconstruction d'une image horizontale et à la reconstruction d'une image verticale. Ce n'est pas une innovation romane. Il est attesté dès l'Antiquité tardive dans les figurations architecturales. La " déconstructionreconstruction " de l'édifice dans l'imagerie tardo-antique, qui apparaît comme une formidable audace conceptuelle et figurative, n'est en fait que l'aboutissement ultime, certes spectaculaire, d'une tendance artistique - aplatissement et restructuration - enclenchée à la charnière des $\mathrm{II}^{\mathrm{e}}$ et $\mathrm{III}^{\mathrm{e}}$ siècles. Les mosaïques et peintures murales de l'Antiquité tardive et de l'art proto-byzantin fournissent un corpus remarquable de figurations architecturales recomposées, qu'il s'agisse de villes, de villa, de palais ou d'églises ${ }^{8}$. Sur la perception et la réception de ces figurations,

7. En 1972, Joergensen Bent avait mis en évidence le phénomène à propos du tympan de Moissac dont on retrouve l'iconographie traitée à l'horizontale dans les pleines pages des Beatus. Il avait montré que les registres superposés des vingt-quatre vieillards correspondaient, sur un plan horizontal, à un cercle de personnages entourant le Christ et que ce cercle, impossible à figurer sur le plan vertical du tympan, avait été découpé en sections distribuées sur le tympan. Joergensen Bent, "La composition du tympan de Moissac expliquée par une projection panoramique ", Cabiers de civilisation médiévale, $15^{\mathrm{e}}$ année, $\mathrm{n}^{\circ}$ 60, octobre-décembre 1972, p. 303-308.

8. Dans la large bibliographie sur le sujet, voir par exemple: Ingrid Ehrenspergberger-Katz, "Les représentations de villes fortifiées dans l'art paleochrétien et leurs dérivées byzantines ", Cahiers Archéologiques, vol. 19, 1969, p. 1-27 ; Adriano Peroni, "Raffigurazione e progettazione di strutture urbane e erchitettoniche nell'Alto Medioevo ", Topografia urbana e vita citadina nell'Alto Medioevo in Occidente, Settimane di studio del Cisam (26 avril-1 $1^{\text {er }}$ mai 1973), t. 2, Spolète, 1974, p. 679710 ; Janine Balty, Mosaïques antiques du Proche-Orient: chronologie, iconographie, interprétation, Paris, Les Belles Lettres, 1978 ; Tadeusz Sarnowski, La Représentation de villas sur les mosaïques africaines, Varsovie, 1978 ; Pauline Donceel-Voûte, "La carte de Madaba : cosmographie, anachronisme et propagande ", Revue biblique, vol. 95, 1988, p. 519-542 ; Noël Duval, "La mosaïque du Palatium de Saint-Apollinaire-le-Neuf représente-t-elle une façade ou un édifice aplani ? ", Corso di Cultura sull'Arte Ravennate e Bizantina, t. XXV, 1978, p. 93-122 ; Johannes Deckers, "Traditio und Adaptatio : Bemerkungen zur Darstellung der christlichen Stadt ", Römisches Mittelalter, vol. 95, 
Noël Duval a donné une clef de lecture pertinente à propos d'une représentation originale, appelée couramment villa maritime ou ville maritime, qui orne le centre d'un plat d'argent du IV siècle retrouvé dans les fouilles de Kaiseraugst (Augusta Raurica), en Suisse actuelle, et dénommé, pour cette raison, plat de Kaiseraugst ${ }^{9}$.

Le médaillon central du plat, un dessin finement gravé et traité au nielle, présente un ensemble de constructions situées au bord d'une mer où foisonnent des animaux marins et des pècheurs ${ }^{10}$. On y distingue trois édifices couverts d'une coupole côtelée comme une sorte de tholos ${ }^{11}$, quatre façades à fronton, quatre murs portant un toit en appentis, difficiles à identifier en tant qu'architecture, et trois colonnades semblablement couvertes, qui suggèrent des portiques. La distribution de ces constructions est réglée sur une symétrie rigoureuse et sur des plans successifs qui créent des effets de profondeur par dissimulation partielle des arrières-plans, mais sans diminution de volume. L'apparence de ville ou de villa ainsi évoquée est toutefois contredite par l'incohérence des détails. Noël Duval a pu restituer de façon très convainquante l'image d'une basilique où sont employés tous les fragments d'architecture décrits plus haut (ill. 1) : abside, murs gouttereaux, colonnades du vaisseau central, façade et toitures. Cette basilique a donc fait l'objet d'un démontage par objets architecturaux représentatifs, d'une démultiplication de ces éléments et d'une dispersion suivant des critères non pas architecturaux, mais iconiques, visant à créer une image nouvelle. Cette démarche est similaire à celle de la description littéraire, plus précisément de cette ekphrasis emphatique propre à l'Antiquité tardive, qui met en valeur les principales composantes architecturales, en les détachant de l'ensemble et en les présentant sous différents angles.

Les illustrations du Psautier d'Utrecht, réalisé sans doute à Reims au IX ${ }^{\mathrm{e}}$ siècle dans la mouvance de la Renaissance carolingienne, reprennent la formule de déconstruction-reconstruction mise en ouvre dans le plat de Kaiseraugst ${ }^{12}$. Mais

1989, p. 303-382 ; Noël Duval, "L'iconographie architecturale dans les mosaïques de Jordanie ", Mosaïques byzantines de Jordanie, cat. expos. Lyon, février-mais 1989, Lyon, 1989, p. 207-214; Michele Piccirillo, The Mosaics of Jordan, Amann, American Center of Oriental Research, 1993; Noël Duval, "Le rappresentazioni architettoniche", Umm Al-Rasas Mayfa'ah, I, Gli scavi del complesso di Santo Stefano, M. Piccirillo et E. Alliata dir., Jérusalem, 1994, p. 165-230 ; Carlo Bertelli, "Visual Images of the Town in late Antiquity and Early Middle Ages ", The Idee and Ideal of the Town between late Antiquity and the Early Middle Ages, $t$. 4: The Transformation of the Roman World, G. Brogiolo et B. Ward-Perkins dir., Leyde, 1999, p. 127-146 ; Isabella Baldini, "Cultura figurativa, dall'età dei Severi alla dinastia costantiniana ", Aemilia, La cultura romana in Aemilia Riomagna dall III secolo a. c. all'età Constantiana, cat expos. Bologne, M. Marini Calvani dir., Venise, 2000, p. 279-287.

9. Noël Duval, «L'architecture sur le plat en argent dit "À la ville maritime" de Kaiseraugst (première moitié du IV e siècle) : un essai d'interprétation ", Bulletin Monumental, 1988, p. 341-353. Pour une bibliographie générale de la question, voir Annemarie Kaufmann-Henimann, "Bilanz der Forschung seit 1984. ", Der spätrömische Silberschatz von Kaiseraugst, Die Neuen Funde, Martin A. Giggisberg dir., Römerstadt Augusta Raurica, 2003, p. 15-30.

10. Les édifices occupent la moitié supérieure du disque, la mer, la moitié inférieure.

11. Deux sont dotées d'une baie d'axe encadrée de deux colonnes, une de trois baies carrées sans décor.

12. Noël Duval, "La représentation du palais dans l'art du Bas-Empire et du haut Moyen Âge d'après le psautier d'Utrecht ", Cahiers Archéologiques, vol. 15, 1965, p. 207-254. Sur le Psautier d'Utrecht, voir : Suzanne Dufrenne, Les Illustrations du Psautier d'Utrecht, Sources et apports carolingiens, Paris, 


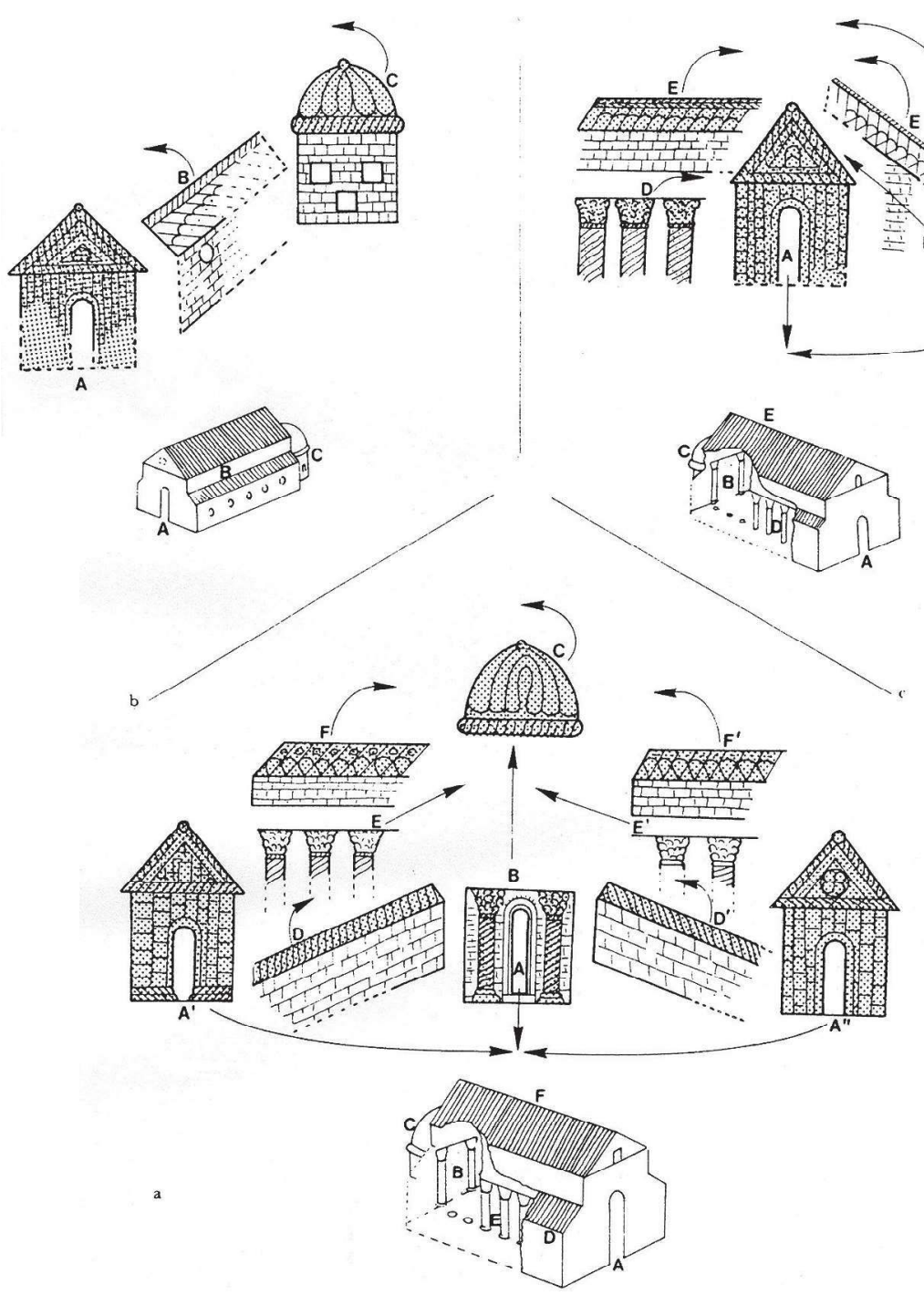

Ill. 1 : Restitution de la basilique du plat de Kaiseraugst par Noël Duval. (Noël Duval, «L'architecture sur le plat en argent dit "À la ville maritime" de Kaiseraugst (première moitié du IV siècle) : un essai d'interprétation ", Bulletin Monumental, 1988, p. 341-353. ( ) Avec l'aimable autorisation de l'auteur.

l'occupation de l'architecture par un groupe de personnages change radicalement le « remontage " de l'édifice. Il ne s'agit pas en effet d'une architecture faisant office

1978 ; The Utrecht Psalter in Medieval Art, Picturing the Psalm of David, cat. expos. Utrecht, Museum Catharijneconvent, 31 août-17 novembre 1996, Koert van der Horst, William Nœl, Wilhelmina C. M. Wüstefeld dir., Westrenen, HES Publishers, 1996. 


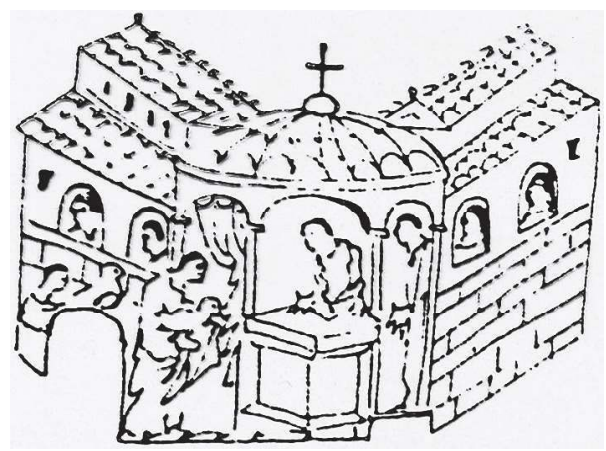

Ill. 2 : La basilique du psaume 28 (29), d'après la figure du Psautier d'Utrecht. Noël Duval, «La représentation du palais dans l'art du Bas-Empire et du haut Moyen Âge d'après le psautier d'Utrecht ", Cahiers Archéologiques, vol. 15, 1965, p. 207-254. (C) Avec l'aimable autorisation de l'auteur.

de décor habité, comme dans les sarcophages païens ou chrétiens de l'Antiquité, mais bien d'une architecture dans laquelle se déroule une scène qui lui est étroitement associée et qui lui impose une restructuration narrative. C'est ce que l'on constate dans le dessin complexe de la basilique (ill. 2) où se tiennent les porteurs d'offrandes du psaume $28(29)^{13}$. La nef à trois vaisseaux est figurée comme deux ailes séparées, divergeant depuis l'abside, comme si l'on pouvait voir la même architecture sous deux angles différents, à la manière du cubisme. En dissociant dans la même figure les deux moitiés de la nef pour en montrer simultanément des deux murs gouttereaux, le dessin reproduit, dans le domaine graphique, la figure littéraire de l'hendiadyn ${ }^{14}$.

L'essentiel de la scène se déroule dans le sanctuaire. Il est figuré, non pas dans son étendue et son volume, mais par un élément architectural clairement identifiable, la triple arcade donnant sur l'espace de l'autel, surmontée - composition a-réaliste - d'un cul de four révélé par la forme de la toiture. Cette réduction de l'espace à un élément identitaire équivaut, dans les figures de style littéraires, à une synecdoque de la partie pour le tout ${ }^{15}$. Les porteurs d'offrande viennent visiblement de l'extérieur, mais entrent par l'arcade latérale du triplet, qui se trouve en

13. Psautier d'Utrecht, Utrecht, Universiteitsbibliotheek, MS Bibl. Rhenotraiectinae I Nr 32 , fo $16 \mathrm{r}^{\circ}$.

14. L'hendiadyn est une figure de style qui désigne une réalité par deux aspects subordonnés l'un à l'autre dans les faits, mais qu'elle dissocie pour recomposer un groupe coordonné, c'est-à-dire à égalité de traitement. Dans l'expression : "Il admire le général et sa victoire », l'hendiadyn associe par parataxe ce qui doit être subordonné dans la formule du substantif et du complément du nom : "Il admire la victoire du général ", ou du substantif et de l'adjectif épithète : "Il admire le général victorieux".

15. La synecdoque est une figure de style qui appartient à la classe des tropes. Elle désigne une réalité, non pas par le terme habituel, mais par un autre mot ayant un rapport de continuité avec celuici. Par exemple, "La voiture a crevé " est une métonymie généralisante qui désigne la roue (c'est elle qui a crevé) par le nom de l'ensemble de la machine. "L'anguille retourne frayer dans la mer des Sargasses » est une synecdoque particularisante qui désigne "les anguilles » (synecdoque du singulier pour le pluriel). 
réalité dans l'église. L'officiant, positionné en arrière de l'autel, les regarde arriver à travers l'arcade centrale, tandis que l'acolyte se tient en arrière de l'autre arcade latérale. La distorsion radicale des espaces n’interdit pas une claire lisibilité de la scène.

\section{Figurations iconiques et figures de style}

Les figurations architecturales du Psautier d'Utrecht font donc un usage raisonné et systématique de certaines figures de style adaptables à l'image. Les figurations romanes transposent elles aussi, mais de manière plus large, l'art littéraire dans les compositions figuratives. Prenons les monstres qui se rencontrent partout dans les arts des XII et XIII ${ }^{e}$ siècles. Ce n'est pas la totalité de l'animal, naturaliste (lion, ours ou serpent) ou fantastique (chimère ou bien griffon), qui exprime la violence, mais certains détails choisis pour leur représentativité - gueule, dents, bec recourbé, griffes, queue fouettante ou préhensible... - qui reçoivent un traitement hypertrophié, selon un processus qui n'est pas sans rappeler la formation du rêve. Il s'agit d'une application originale à l'image de la synecdoque (pars pro toto). Dans un tout autre registre, la décollation d'un saint fait le plus souvent appel à l'hendiadyn. La représentation le plus fréquente de la scène montre en effet d'un côté le bourreau l'épée levée, de l'autre, le saint décapité, la tête roulant au sol. Il y a là une incohérence chronologique, puisque l'épée doit s'abattre sur le cou de la vicitme, avant que sa tête ne soit détachée. Cet hendiadyn iconique représente un seul acte - ici, une mise à mort - en deux moments distincts, mais immédiatement consécutifs. Ce qui a commandé le choix de ces deux moments, c'est le caractère éminemment spectaculaire de chacun. À l'inverse, la décapitation plus réaliste qui caractérise souvent la mise à mort de saint Thomas de Cantorbéry montre l'épée déjà abattue sur le cou de l'évêque, mais ne faisant encore qu'entamer les chairs ${ }^{16}$.

L'ampleur du phénomène, encore peu étudié, apparaît avec évidence dans un programme de six chapiteaux de la fin du XII e siècle, distribués dans l'abside de la cathédrale de Lyon, en arrière de l'autel, et consacrés à l'enfance de Jésus ${ }^{17}$. Du côté nord, les trois chapiteaux sont occupés chacun par un roi mage à cheval, forme d'anaphore iconique proche du style épique ${ }^{18}$. Chaque roi mage illustre un épisode du voyage à Béthleem et représente donc chaque fois les trois rois : il s'agit d'une synecdoque (synecdoque du nombre). Le second roi figure la visite chez Hérode qui, absent du chapiteau, est remplacé par une figure diabolique, inspiratrice du mauvais conseil : le diable est donc une métonymie (métonymie de la cause pour

16. L'iconographie du martyre de saint Thomas de Cantorbéry est très diverse. On rencontre principalement la frappe d'estoc, la décollation classique (hendiadyn) ou bien la représentation plus réaliste mentionnée ici.

17. Le septième chapiteau, portant le buste du Christ Pantocrator, timbré sur le tailloir de la formule " ego sum qui sum ", est situé dans l'axe de l'abside et fournit une clef de lecture de l'ensemble.

18. L'anaphore est une figure de style par répétition, qui crée un effet de rythme : le même mot commence des vers ou des phrases successives composant ensemble une unité de sens. 
l'effet) renvoyant au personnage du mauvais roi ${ }^{19}$. Grâce à cette construction dynamique, et très savante, des réseaux de sens sont tissés d'une part entre tous les éléments du programme, d'autre part entre le programme et l'action liturgique se déroulant à l'autel ${ }^{20}$.

\section{Figures de style et figurations architecturales}

La formule de "déconstruction-reconstruction" n'est possible que dans le contexte d'une pensée, ou plutôt d'une perception intuitive de l'architecture, qui substitue spontanément à la dynamique architectonique la subdivision de l'édifice en éléments finis : l'architecture prévaut sur la construction. Les figurations romanes de destruction procèdent toujours selon cette logique ${ }^{21}$. Dans la Bible de Lobbes datée de 1084, pour ne prendre que cet exemple, l'initiale $\mathrm{V}$ du Livre de Jérémie montrant le prophète se lamentant sur les ruines de la ville ${ }^{22}$ figure les ruines d'une ville sous la forme d'éléments architecturaux épars et baculés : une tour, une toiture, deux arcades (ou des portes), des chapiteaux, des colonnes brisées, des blocs d'opus quadratum... Si les fûts cassés de colonnes et les blocs éparpillés appartiennent pleinement à l'image romane de la ruine, l'intégrité de la tour ou des arcades en position de chute est irréaliste, mais évocatrice. En règle générale, la représentation de hauts édifices détruits montre des sections d'architecture tombées au sol (toiture ou étages), chacune restant toutefois intacte et soigneusement délimitée dans ses formes architecturales préservées. Il faut attendre le vérisme gothique pour voir apparaittre des figurations d'architecture ruiniformes, comme l'effondrement de la synagogue progressant de page en page dans le calendrier du Bréviaire de Belleville peint par Jean Pucelle ${ }^{23}$ vers 1323-1326, les villes détruites de la Tenture de l'Apocalypse d'Angers (vers 1380) ou le château ruineux du folio 232 des Chroniques de Hainaut (vers 1448) par exemple ${ }^{24}$.

19. La métonymie est une figure de style qui appartient à la classe des tropes. Elle désigne une réalité, non pas par le terme habituel, mais par un autre mot ayant un rapport logique avec celui-ci. " Boire un verre ", par exemple, est une métonymie du contenant (le verre) pour le contenu (le liquide) et signifie "boire le liquide contenu dans le verre ". Le "verre " lui-même est une métonymie de la matière pour l'objet et signifie : "un gobelet en verre".

20. Nicolas Reveyron, "Image performative et liturgie, les sept chapiteaux de l'abside de la cathédrale de Lyon $\left(\mathrm{XII}^{\mathrm{e}}\right)$ ", L'Église, lieu de performances, In locis competentibus, D. Daussy dir., Paris, Picard, 2016, p. 301-313.

21. Pour une approche de la perception des ruines, voir en dernier lieu Alain Schnapp, Ruines, Essai de perspective comparée, Lyon, PUL, 2015.

22. Bibliothèque du séminaire de Tournai, Cod. 1, Biblia sacra, dite "Bible de Lobbes", fo $195 \mathrm{v}^{\circ}$. Jacqueline Leclerc-Marx, Noémi Thys, "Les initiales historiées, Quelques hypothèses et apports nouveaux ", Autour de la Bible de Lobbes (1084), Les institutions. Les hommes. Les productions, Cahiers du Centre de recherches en histoire du droit et des institutions, vol. 28, 2007, p. 169-209.

23. Bréviaire de Belleville, Paris, Bibliothèque nationale de France, ms lat. 10483.

24. Chroniques de Hainaut I, Bruxelles, Bilbiothèque royale, ms 9242, fo 232. 
Cette représentation mentale de l'architecture et l'intégration de principes de la rhétorique (principalement la synecdoque de la partie pour le tout) dans la construction iconique ont ouvert un vaste champ de possibilités dans la création d'images d'architecture, depuis sa plus simple expression, réduite à quelques éléments schématiques, jusqu'à des compositions complexes équivalent à un véritable discours ecclésiologique. Le vitrail de saint Pierre qui orne la chapelle nord du chouur de la cathédrale de Lyon daté de la fin du XII ${ }^{\mathrm{e}}$ siècle ${ }^{25}$ illustre les possibilités narratives que contient la figuration architecturale le plus simple (ill. 3). Parmi les cinq panneaux ${ }^{26}$, trois médaillons centraux consécutifs sont structurés par la même architecture réduite à une colonne, deux arcs et deux murs latéraux ${ }^{27}$. La répétition de la formule équivaut à une anaphore qui a pour finalité de rythmer le récit, de donner une unité formelle aux scènes centrales et de mettre en évidence, par contraste, les variations de détail. Dans le premier des trois médaillons (la résurrection de Tabitha à Joppé par saint Pierre), la formule est complétée, en contrebas, par un long mur rejoignant les deux murs latéraux, mais réduit à quelques assises pour qu'il ne dissimule pas les scènes du médaillon; ce troisième mur suggère la salle où vivent les veuves et gît Tabitha. Dans le second médaillon (un roi, accompagné d'un conseiller, donne à un soldat l'ordre d'exécuter les deux saints), le mur latéral droit, du côté du soldat qui tourne déjà les talons, est remplacé par une seconde colonne : le palais est grand ouvert sur l'extérieur. Le troisième médaillon, chargé en personnage (martyre de Pierre et de Paul), reprend la formule de base, pour offir à chaque martyr un cadre architectural qu'il serait vain de vouloir identifier : la présence du roi désigne simplement le lieu du pouvoir comme lieu de l'action, pour confirmer la responsabilité morale du donneur d'ordre.

De fait, dans un récit ou dans une expositon dogmatique, la figure architecturale ne se comprend pas seule, mais dans son contexte, soit structurel, comme dans le vitrail de saint Pierre, soit narratif. Ainsi, dans le tympan de Sainte-Foy de Conques par exemple ${ }^{28}$, la représentation à dextre du paradis, avec la figure d'Abraham, est composée simplement d'une suite d'arcades allant diminuant depuis le centre et du faîte de deux tours crénelées. La scène se pose comme l'antithèse de celle de l'enfer, située à senestre : la première est une mise en ordre et une protection des ressuscités par l'architecture, la seconde est l'expression d'un désordre par absence d'architecture. À l'opposé de ces figurations simples, la représentation dans une miniature du Manuscrit de Saint-Martin-des-champs ${ }^{29}$ de la consécration de l'autel majeur de Cluny III par le pape Urbain II illustre la complexité de la

25. Catherine Brisac, "Lyon, cathédrale Saint-Jean", Les Vitraux de Bourgogne, Franche Comté et Rhône-Alpes, III, Corpus Vitrearum, Paris, Inventaire général, 1986, p. 293-301.

26. Le premier médaillon central représente saint Pierre accompagnant le Christ marchant sur les eaux du lac de Génésareth et le dernier, le Christ de la Traditio clavis et legis.

27. Réduits à deux empilements étroits de pierres de taille.

28. Jean-Claude Bonne, L'Art roman de face et de profil, le tympan de Conques, SFIED, 1984.

29. Paris, Bibliothèque nationale de France, ms 17716, fo $91 \mathrm{r}^{\circ}$. 


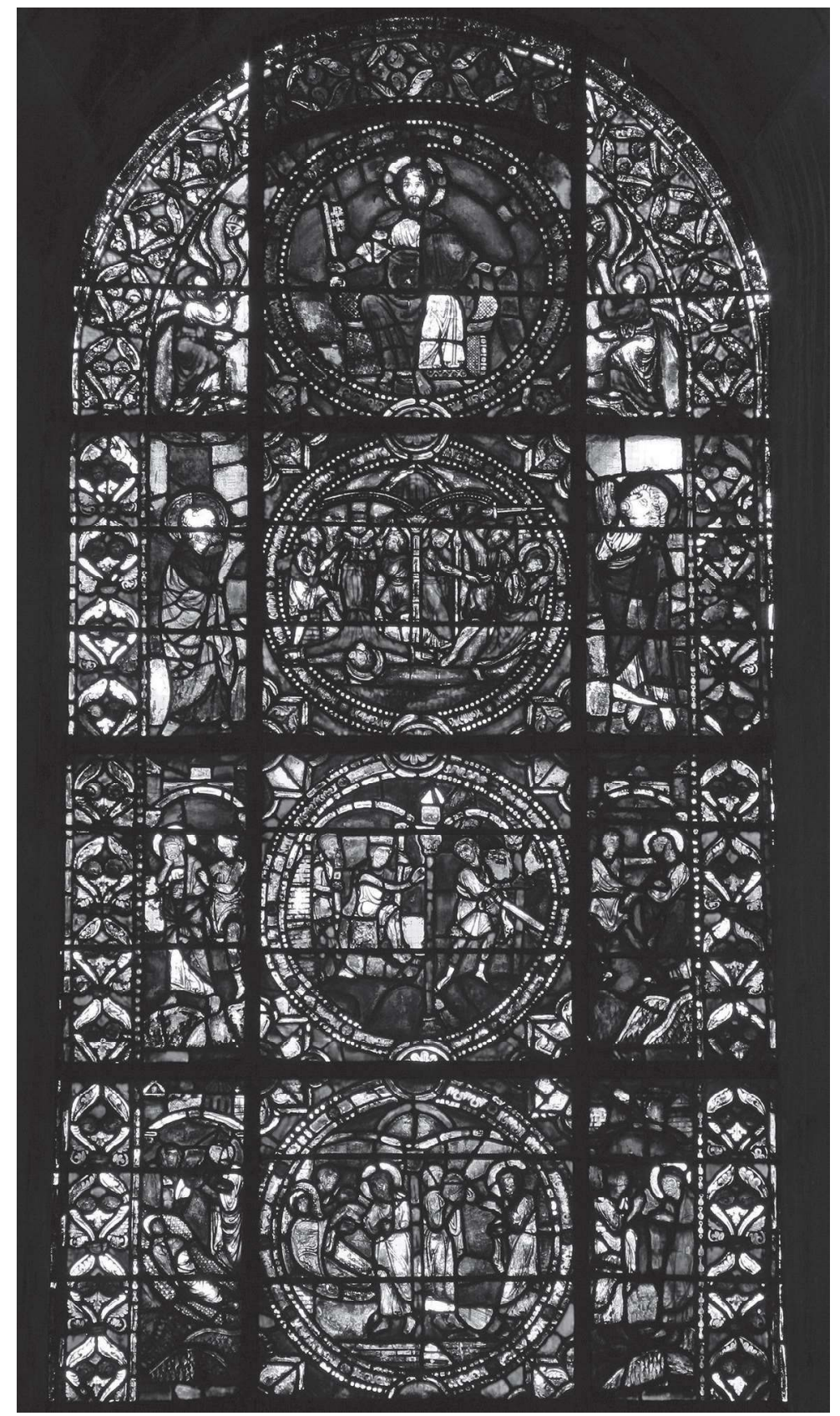

Ill. 3 : Lyon, cathédrale Saint-Jean, vitrail de Saint-Pierre, fin XII siècle. @ C Cliché J.-P. Gobillot. 


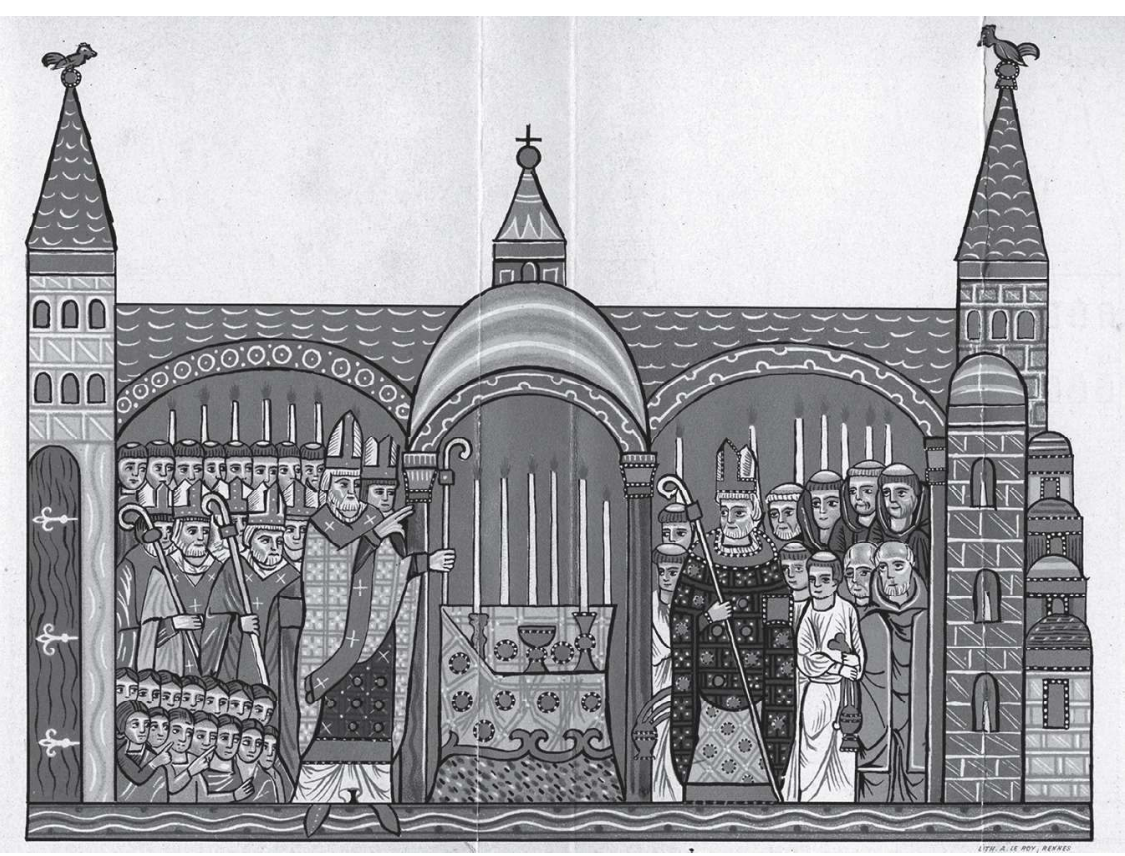

Ill. 4 : Consécration de l'autel majeur de Cluny III par le pape Urbain II, Paris, Bibliothèque nationale de France, ms 17716, folio 91 recto. Reproduction par chromolithographie d'après dom A. L'Huillier, Vie de saint Hugues, sixième abbé de Cluny, Solesmes, Imprimerie Saint-Pierre, 1888.

composition et de ses significations (ill. 4) ${ }^{30}$. L'édifice est identifiable de profil, c'est-à-dire sur un axe est-ouest, disposition qui met en valeur la longueur de l'abbaye ainsi que ses tours. À gauche, une grande tour dotée d'une haute porte traduit classiquement l'entrée de l'église. À droite, au-delà d'une autre tour (tour lanterne ?), une sorte de grande tour cylindrique et coiffée d'une demi-sphère est accolée de quatre petites tours semblables: il s'agit de l'abside principale et des absidioles, dont les fenêtres laissent voir les flammes des cierges qui brillent dans le sanctuaire. L'intérieur, cependant, matérialisé par trois arcs, se développe sur un axe nord-sud : le pape, avec la curie, à gauche, Hugues de Semur et le convent, à

30. Brigitte Maurice dir., Cluny III, la Major Ecclesia, Musée Ochier et ville de Cluny, 1988, notice 131, p. 116-118 ; W. Kahn, Romanesque Manuscripts, The Twelth Century, Londres, Harvey Miller, 1996, t. II, n 93, p. 114-115; Éric Palazzo, L'Évêque et son image, L'illustration du pontifical au Moyen Âge, Turnhout, Brepols, 1999, p. 349-351 ; Dominique Iogna-Prat, "Introduction, Ecclésiologie et système ecclésial clunisien ", Études clunisiennes, Paris, Picard, 2002, p. 11-34; Didier Mehu, "Un monument de l'historiographie clunisienne du XIII e siècle : le manuscrit Paris, BnF, Latin 17716 ", Cluny ou la puissance des moines, Histoire de l'abbaye et de son ordre, 910-1790, Dossiers d'archéologie, $\mathrm{n}^{\circ} 269$, janvier 2002, p. 56 ; Dominique Iogna-Prat, "Un recueil liturgique et historique du tournant des années 1200 (Paris, BnF, ms latin 17716)", Bulletin du Centre d'études médiévales d'Auxerre, vol. 9, 2005 (http: //cem.revues.org/document792.html). 
droite. Cette distorsion permet, d'une part de distribuer les groupes de chaque côté de l'autel suivant une étonnante égalité, d'autre part, comme l'avait déjà noté Patricia Stirnemann, de tisser les colonnes de texte avec l'image, celle de gauche étant consacrée au pape, celle de droite, à Hugues de Semur ${ }^{31}$. C'est bien l'ecclésiologie clunisienne qui est traitée dans cette image restructurée.

\section{Conclusion}

Ces quelques exemples présentés rapidement montrent déjà toute la richesse des représentations romanes de l'architecture, héritières de l'Antiquité. Les modalités de composition des images architecturales empruntent aux figures de style de la rhétorique classique, parce qu'elles visent le même but expressif. Elles ne sont pas figées, mais règlent les figurations en fonction de leur situation et des contextes. Elles répondent aussi à des exigences de communication. L'inventivité exceptionnelle révélée par certaines pièces, comme la consécration de l'autel majeur de Cluny III par le pape Urbain II, déborde du seul domaine de l'image pour intéresser aussi le texte - quoique rare, la tendance est connue depuis le De laudibus Sanctae Crucis de Raban Maur - et le livre. C'est donc une tout autre dimension de l'image qui est ici mise en évidence.

Nicolas ReVEYRON

professeur d'histoire de l'art à l'université Lumière-Lyon 2

31. Patricia Stirnemann, "Un recueil de pièces glorifiant Cluny et son histoire ", Cluny, onze siècles de rayonnement, Neil Stratford dir., Paris, Éd. du Patrimoine, 2010, p. 276-279. 